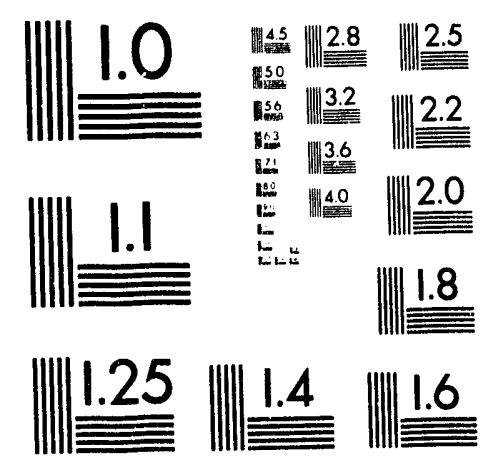



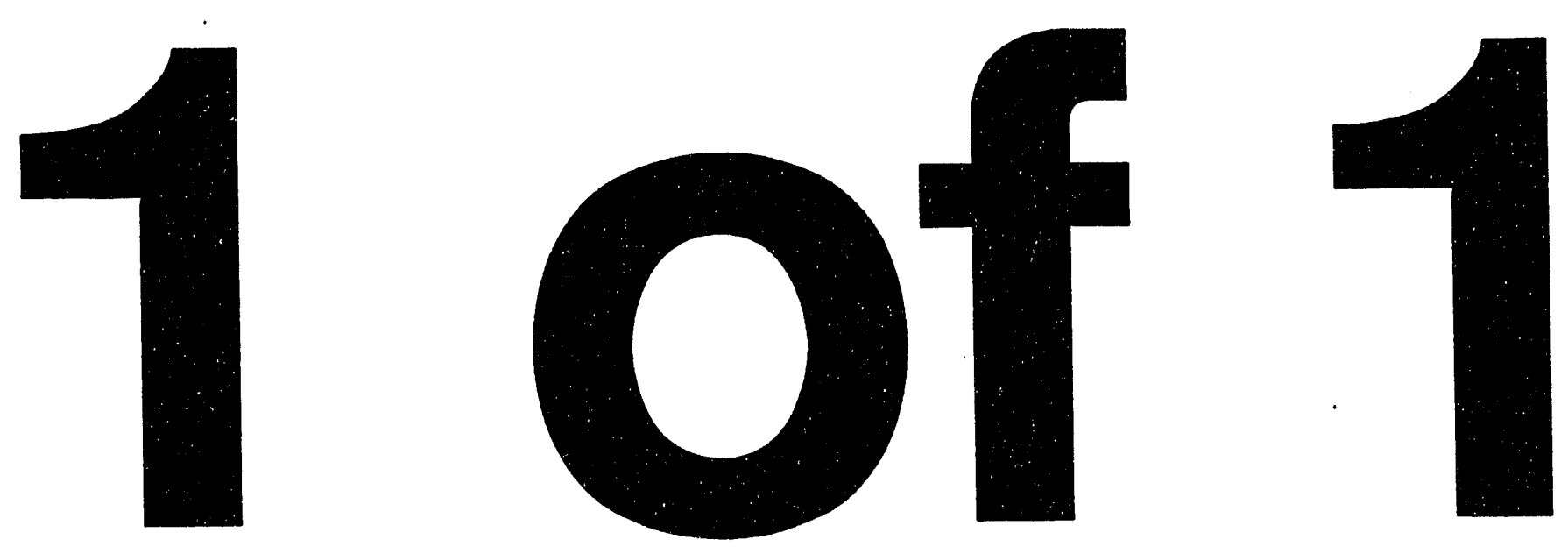
LBL-33288

UC-419

HIFAN-573

\section{Longitudinal Instability of an Induction \\ Linac With Acceleration}

L. Smith and E. P. Lee

Lawrence Berkeley Laboratory

University of California

Berkeley, California 94720

Submitted to the 1993 Particle Accelerator Conference

Washington, D. C., May 17-20, 1993

*This work was supported by the Office of Energy Research, Office of Fusion Energy, U.S. Department of Energy under Contract No. DE-AC03-76SF00098. 


\title{
Longitudinal Instability of an Induction Linac with Acceleration*
}

\author{
Lloyd Smith and Edward P. Lee \\ Lawrence Berkeley Laboratory \\ Berkeley, Califomia 94720 USA
}

\section{Abstract}

The question arises as to what effect acceleration, which so far has been ignored, has on the longitudinal instability of an induction linac. The answer is not much for the anticipated acceleration rate $(1-2 \mathrm{MeV} / \mathrm{m})$ and minimum efolding distance for the instability (50 - 500 meters). However, total unstable growth is significantly reduced over distances which are long enough for appreciable acceleration to occur. The purpose of this note is to record a calculation of the instability, including a constant acceleration rate. Some interesting features emerge - for example, the velocity of the head is a more convenient independent variable than axial position and, for an initial sinusoidal perturbation of velocity in time, the number of oscillations along the pulse is constant; as the pulse shortens in time the frequency increases.

\section{BASIC EQUATIONS AND UNPERTURBED SOLUTION}

We start, as in previous work, with the one-dimensional cold fluid equations, neglecting the space charge force, and adopting a parallel $\mathrm{R}-\mathrm{C}$ circuit for the perturbed electric field from the induction modules:

$$
\begin{aligned}
& \partial \lambda / \partial t+\partial \mathrm{I} / \partial z=0 \\
& \partial v / \partial t+v \partial v / \partial z=E_{0}+E, \\
& \partial E / \partial t+E / \tau=-e \Delta I / m C,
\end{aligned}
$$

where $E_{0}$ is the applied field (multiplied by $\mathrm{e} / \mathrm{m}$ ), $\mathrm{C}$ is the circuit capacity and $\tau=R C$. The circuit parameters, $R$ and $C$, might in general depend on axial position. $E$ and $\Delta I$ are the perturbed components of electric field and beam current, while $I, \lambda$, and $v$ are total beam current line charge and velocity.

The velocity of the head is given by:

$$
v_{\mathrm{H}}^{2}=\mathrm{v}_{\mathrm{i}}^{2}+2 \mathrm{az}
$$

\footnotetext{
*This work supported by the Director, Office of Energy Research, Office of Fusion Energy, of the U.S. Department of Energy under Contract No. DE-AC03-76SF00098.
}

where $a$ is the constant acceleration and $v_{i}$ is the head velocity at $z=0$, where the initial perturbation occurs.

We change the independent variables $(z, t)$ to $\bar{z}=\mathrm{z}$ and:

$$
\bar{t}=\mathrm{t}-\int_{0}^{\mathrm{z}} \frac{\mathrm{dz}}{\mathrm{v}_{\mathrm{H}}},
$$

Eliminating $\lambda$, the fluid equations become:

$$
\begin{aligned}
& \frac{\partial}{\partial \bar{t}}\left(\frac{1}{v_{i}}-\frac{1}{v_{H}}\right) I+\frac{\partial I}{\partial \bar{z}}=0, \\
& v\left(\frac{1}{v}-\frac{1}{v_{H}}\right) \frac{\partial v}{\partial \bar{t}}+v \frac{\partial I}{\partial \bar{z}}=E_{O}+E, \\
& \frac{\partial E}{\partial \dot{\tau}}+\frac{E}{\tau}=-\frac{e \Delta I}{m C} .
\end{aligned}
$$

\section{CASE OF INCREASING CURRENT}

For the unperturbed pulse, we take $I=v_{H} I_{i} / v_{i}$, independent of time during the pulse and lasting for a time, $T=v_{i} T_{i} / v_{H}$. The velocity during the pulse is given by (1'):

$$
v=\frac{v_{H}}{1-\frac{\mathrm{at}}{v_{H}}} .
$$

Note that a $T / v_{H}$ is the velocity tilt $\Delta \beta / \beta$. In the GeV energy range, this quantity is quite small. The required voltage wave shape as a function of $z$ and $t$ is given by $\left(2^{\prime}\right)$ :

$$
E_{0}=a \frac{\left(1-\frac{3 a \bar{t}}{v_{H}}\right)}{\left(1-\frac{a \bar{t}}{v_{H}}\right)^{3}}=a\left[1-3\left(\frac{a \bar{t}}{v_{H}}\right)^{2}\right] \text {. }
$$

\section{INSTABILITY}

The next step is to find equations for a linearized perturbation of the unperturbed quantities. This process is rather messy because the unperturbed quantities also depend 
on $\bar{z}$ and $\bar{t}$. However, the equations are greatly simplified by replacing $\overline{\mathrm{z}}$ and $\overline{\mathrm{t}}$ by $\mathrm{v}_{\mathrm{H}}$ and a scaled time:

$$
\begin{aligned}
& v_{H}=\sqrt{v_{i}^{2}+2 a \bar{z}}, \\
& t^{\prime}=\frac{v_{H}}{v_{i}} \bar{t},
\end{aligned}
$$

and taking the perturbed quantities to be of the form:

$$
\begin{aligned}
& I+\Delta I=\frac{v_{H}}{v_{i}} I_{i}(1+u), \\
& \Delta v=\left(1-\frac{a \bar{t}}{v_{H}}\right) \hat{v} .
\end{aligned}
$$

The equations for $\mathrm{u}, \widehat{\mathrm{v}}$, and $\mathrm{E}$ are:

$$
\begin{aligned}
& \frac{\partial u}{\partial v_{H}}=\frac{1}{a v_{i}} \frac{\partial}{\partial t^{\prime}}\left(1-\frac{a v_{i}}{v_{H}^{2}} t^{\prime}\right)^{3} \hat{v}, \\
& \frac{\partial \hat{v}}{\partial v_{H}}=\frac{1}{a} E, \\
& \frac{\partial E}{\partial t^{\prime}}+\frac{v_{i}}{v_{H}} \frac{E}{\tau}=-k^{2} v_{i}^{3} u, \\
& k^{2}=\frac{e I_{i}}{m C v_{i}^{3}} .
\end{aligned}
$$

These equations are of the same form as those for the unaccelerated case except for the cubed parenthesis in (10) and the factor, $\mathrm{v}_{\mathrm{i}} / \mathrm{v}_{\mathrm{H}}$ in (12). The equations are exact in the sense that $\mathrm{a} / \mathrm{v}$ is not yet assumed to be small. The duration in $t^{\prime}$ is:

$$
T^{\prime}=\frac{v_{H}}{v_{i}} \bar{T}=T_{i}
$$

which means that a disturbance maintains the same relative position in time as the pulse shortens and the rate of change in real time increases. In particular, a sinusoidal perturbation would retain the same number of cycles over the pulse duration, as mentioned above.

Equation (12) is simplified if we assume that the gap capacity is independent of position but that the matched resistance is inversely proportional to the current (directly proportional to pulse duration). Equation (12) is then:

$$
\partial E / \partial t^{\prime}+E / \tau_{i}=-k^{2} v_{i}^{3} u \text {, }
$$

where $\tau_{\mathrm{i}}$ is the time constant at $z=0$.
Furthermore, the extra terms in the cubed parenthesis in (10) appear to give corrections of order $\Delta \beta / \beta$ and can be neglected. The equations then have the same form as for the unaccelerated case; if the initial perturbation is :

$$
\widehat{v}(z=0)=\delta e^{i \omega t}
$$

a particular solution for $\widehat{\mathrm{v}}$ is:

$$
\begin{aligned}
\hat{v} & =\delta e^{i \omega x^{\prime}} \cos \left[\frac{k v_{i}}{a} \sqrt{\frac{i \omega \tau_{i}}{1+i \omega \tau_{i}}}\left(v_{H}-v_{i}\right)\right] \\
& =\delta e^{i \omega \frac{\eta_{H}}{v_{i}}} \cos \left[k z \sqrt{\frac{i \omega \tau_{i}}{1+i \omega \tau_{i}}} \frac{2 v_{i}}{v_{H}+v_{i}}\right] .
\end{aligned}
$$

For $\mathrm{a}=0$, we have $\mathrm{v}_{\mathrm{i}}=\mathrm{v}$ and the e-folding rate with $\mathrm{z}$ is the imaginary part of $\sqrt{i \omega \tau /(1+i \omega \tau)} \mathrm{k}$. The maximum growth rate is $k / \sqrt{8}$, which occurs when $\omega \tau=1 / \sqrt{3}$. For $a \neq$ 0 and $z$ large, $v_{H} \sim \sqrt{2 a z}$ and the perturbation only grows exponentially with $z^{1 / 2}$. However, if $k \sim(50 \text { meters })^{-1}$, the accelerating gradient - $1 \mathrm{MeV} /$ meter and a perturbation occurs at $~ 1 \mathrm{GeV}$, there are many e-foldings before $v_{H}$ is significantly greater than $v_{\mathbf{i}}$. The coasting beams assumption is then good enough to show the nature of the problem. however it is of interest to examine the breakdown of this approximation over long distances.

\section{CASE OF CONSTANT CURRENT}

So far we have computed the instability growth rate for a pulse where the current increases proportional to $v_{H}(z)$, i.e. approximately fixed pulse length in meters. It is also of interest to compute growth for the case of constant current, where pulse length increases proportional to $v_{H}$ but pulse duration $T$ is constant. This could be the preferred approach at high energy if a practical lower limit on pulse duration for the synthesis of acceleration waveforms is observed (say $T$ > $100 \mathrm{~ns}$ ). In this case the unperturbed velocity of the entire pulse is $v(z)=v_{H}(z)=\sqrt{v_{i}^{2}+2 a z}$, and in place of eqns. $\left(1^{\prime}\right)-\left(3^{\prime}\right)$ we have for the perturbed components $\Delta v, \Delta I, E$ :

$$
\begin{aligned}
& v^{2} \partial \Delta \mathrm{I} / \partial \overline{\mathrm{z}}=\partial \Delta \mathrm{v} / \partial \overline{\mathrm{t}}, \\
& \partial(\mathrm{v} \Delta \mathrm{v}) / \partial \overline{\mathrm{z}}=\mathrm{E}, \\
& \partial \mathrm{E} / \partial \overline{\mathrm{t}}+\mathrm{E} / \tau=-\mathrm{e} \Delta \mathrm{I} / \mathrm{mC} .
\end{aligned}
$$

Since $E_{0}$ and $I$ are both constant it is reasonable to assume $R$ and $C$ are separately constant, since for efficient energy transfer we scale

$$
\mathrm{R} \propto \mathrm{E}_{\mathrm{o}} / \mathrm{I} \text { and } \mathrm{RC}=\tau \propto \mathrm{T} \propto \mathrm{I}^{-1}
$$


With these assumptions eqns. (1" - 3") yield an equation for $\Delta \mathrm{v}$ :

$$
\left(\frac{\partial}{\partial \bar{t}}+\frac{1}{\tau}\right) \frac{\partial^{2}}{\partial \bar{z}^{2}}(v \Delta v)=-\frac{\mathrm{eI}}{\mathrm{mC}} \frac{1}{\mathrm{v}^{3}} \frac{\partial}{\partial \bar{t}}(\mathrm{v} \Delta \mathrm{v})
$$

Taking an initial perturbation, $\Delta v=\delta e^{i \omega t}$, with $\omega$ real, we have

$$
\frac{\partial^{2} v \Delta v}{\partial \bar{z}^{2}}=-k^{2} \frac{i \omega \tau}{1+i \omega \tau}\left(\frac{v_{i}}{v}\right)^{3}(v \Delta v)
$$

where $\mathbf{k}$ is given by eqn. (13).

In general, eqn. (16) is solved by the bessel functions $J_{2}$ and $\mathrm{N}_{2}$ of the argument $\mathrm{x}$ :

$$
x=k \sqrt{\frac{i(\omega \tau}{1+i \omega \tau}} \frac{2 v_{i}^{3 / 2}}{a}\left(\sqrt{v}-\sqrt{v_{i}}\right) .
$$

A convenient asymptotic form, obtained by the WKB method, is

$$
\Delta v=\delta e^{i \bar{x}^{-}}\left(\mathrm{v}_{\mathrm{i}} / \mathrm{v}\right)^{1 / 4} \cos (\mathrm{x})
$$

Again, for small $z$ growth is identical with that of the coasting beam. However, for large $z$ a perturbation increases exponentially with $z^{1 / 4}$. This reduced rate of growth reflects the dilution of line charge density during acceleration at constant current. Note that the number of wavelength within the pulse $(=\omega \mathrm{T} / 2 \pi)$ remains constant, as before.

\section{SUMMARY}

In order to compare growth formulas for the three cases we have examined (drifting beam, constant line charge density, and constant current), it is convenient to define a scale length proportional to $v_{i}^{2}$ :

$$
z_{0}=v_{i}^{2} / 2 a, v_{H}=v_{i} \sqrt{1+z / z_{0}}
$$

Coasting Beam: $\Delta v \propto \cos k z \sqrt{\frac{i \omega \tau}{1+i \omega \tau}}$,

\section{Current proportional to v:}

$$
\Delta v \propto \cos \left[k z \sqrt{\frac{i \omega \tau}{1+i \omega \tau}} \frac{2}{1+\left(1+z / z_{0}\right)^{1 / 2}}\right]
$$

\section{Constant Current:}

$$
\begin{gathered}
\Delta v \propto\left(\frac{1}{1+z / z_{0}}\right)^{1 / 8} \cos \left[k z \sqrt{\frac{i \omega \tau}{1+i \omega \tau}} \frac{2}{1+\left(1+z / z_{0}\right)^{1 / 2}}\right. \\
\left.\cdot \frac{2}{1+\left(1+z / z_{0}\right)^{1 / 4}}\right] .
\end{gathered}
$$

Keeping in mind that $\mathrm{z}_{0}$ will be in the range $100 \mathrm{~m}$ $2000 \mathrm{~m}$, the following table gives the predicted reductions in the exponential of growth.

Note that for short distances of a few hundred meters ( $z$ $\leq z_{0}$ ) the coasting beam growth rate is an adequate approximation. At most, a few e-folds of growth are expected in this distance, and feedforward correction might be applied to eliminate further growth. However, when long distances ( $z$ $\gg z_{0}$ ) without use of corrections are considered, it is seen from Table I that very substantial reductions of total sirowth are predicted.

\section{REFERENCES}

1) Edward Lee, Proc. 1997 Linear Accelerator Conference at Ottawa, Ontario, Canada, AECL-10728, Vol. 2, pg. 591.

\begin{tabular}{|c|c|c|c|}
\hline \multicolumn{2}{|c|}{$I \propto v$} & $I=$ constant \\
\hline 0.00 & $\frac{v}{z_{0}}=\sqrt{1+\frac{z}{z_{0}}}$ & $\frac{2}{\left(1+v_{H} / v_{i}\right)}$ & $\frac{4}{\left(1+v_{H} / v_{i}\right)\left(1+\sqrt{v_{H}} / v_{i}\right)}$ \\
\hline 0.30 & 1.0000 & 1.0000 & 1.0000 \\
\hline 1.00 & 1.1402 & 0.9345 & 0.9038 \\
\hline 3.00 & 1.4142 & 0.8284 & 0.8013 \\
\hline 10.00 & 2.0000 & 0.6677 & 0.5523 \\
\hline 30.00 & 3.3166 & 0.4633 & 0.3285 \\
\hline 5.5678 & 0.3045 & 0.1813 \\
\hline
\end{tabular}

Table 1. Reduction of growth with accelcration for current proportional to velocity and constant current. The tabulated factor is the reduction of exponential growth rate compared with that of a coasting bcam. 
With these assumptions eqns. (1" - 3") yield an equation for $\Delta \mathrm{v}$ :

$$
\left(\frac{\partial}{\partial \dot{t}}+\frac{1}{\tau}\right) \frac{\partial^{2}}{\partial \bar{z}^{2}}(v \Delta v)=-\frac{e I}{m C} \frac{1}{v^{3}} \frac{\partial}{\partial \bar{t}}(v \Delta v)
$$

Taking an initial perturbation, $\Delta \mathrm{v}=\delta \mathrm{e}^{\mathrm{i \omega t}}$, with $\omega$ real, we have

$$
\frac{\partial^{2} v \Delta v}{\partial \bar{z}^{2}}=-k^{2} \frac{i \omega \tau}{1+i \omega \tau}\left(\frac{v_{i}}{v}\right)^{3}(v \Delta v)
$$

where $\mathrm{k}$ is given by eqn. (13).

In general, eqn. (16) is solved by the bessel functions $\mathrm{J}_{2}$ and $\mathrm{N}_{2}$ of the argument $\mathrm{x}$ :

$$
x=k \sqrt{\frac{i \omega \tau}{1+i \omega \tau}} \frac{2 v_{i}^{3 / 2}}{a}\left(\sqrt{v}-\sqrt{v_{i}}\right) .
$$

A convenient asymptotic form, obtained by the WKB method, is

$$
\Delta \mathrm{v} \approx \delta \mathrm{e}^{\mathrm{i} \omega \overline{\bar{x}}}\left(\mathrm{v}_{\mathrm{i}} / \mathrm{v}\right)^{1 / 4} \cos (\mathrm{x})
$$

Again, for small $z$ growth is identical with that of the coasting beam. However, for large $z$ a perturbation increases exponentially with $z^{1 / 4}$. This reduced rate of growth reflects the dilution of line charge density during acceleration at constant current. Note that the number of wavelength within the pulse $(=\omega \mathrm{T} / 2 \pi)$ remains constant, as before.

\section{SUMMARY}

In order to compare growth formulas for the three cases we have examined (drifting beam, constant line charge density, and constant current), it is convenient to define a scale length proportional to $v_{i}^{2}$ :

$$
\mathrm{z}_{\mathrm{o}}=\mathrm{v}_{\mathrm{i}}^{2} / 2 \mathrm{a}, \mathrm{v}_{\mathrm{H}}=\mathrm{v}_{\mathrm{i}} \sqrt{1+\mathrm{z} / \mathrm{z}_{\mathrm{o}}},
$$

Coasting Beam: $\Delta v \propto \cos k z \sqrt{\frac{i \omega \tau}{1+i \omega \tau}}$,

\section{Current proportional to v:}

$$
\Delta v \propto \cos \left[k z \sqrt{\frac{i \omega \tau}{1+i \omega \tau}} \frac{2}{1+\left(1+z / z_{0}\right)^{1 / 2}}\right]
$$

Constant Current:

$$
\begin{gathered}
\Delta v \propto\left(\frac{1}{1+z / z_{0}}\right)^{1 / R} \cos \left[k z \sqrt{\frac{i \omega \tau}{1+i \omega \tau}} \frac{2}{1+\left(1+z / z_{0}\right)^{1 / 2}}\right. \\
\left.\cdot \frac{2}{1+\left(1+z / z_{0}\right)^{1 / 4}}\right] .
\end{gathered}
$$

Keeping in mind that $z_{0}$ will be in the range $100 \mathrm{~m}$ $2000 \mathrm{~m}$, the following table gives the predicted reductions in the exponential of growth.

Note that for short distances of a few hundred meters ( $z$ $\leq z_{0}$ ) the coasting beam growth rate is an adequate approximation. At most, a few e-folds of growth are expected in this distance, and feedforward correction might be applied to eliminate further growth. However, when long distances ( $z$ $\gg z_{0}$ ) without use of corrections are considered, it is seen from Table I that very substantial reductions of total growth are predicted.

\section{REFERENCES}

1) Edward Lee, Proc. 1997 Linear Accelerator Conference at Ottawa, Ontario, Canada, AECL-10728, Vol. 2, pg. 591.

\begin{tabular}{|c|c|c|c|}
\hline \multicolumn{2}{|c|}{$I \propto v$} & $I=$ constant \\
\hline 0.00 & $\frac{\mathrm{z}}{\mathrm{z}_{\mathrm{i}}}=\sqrt{1+\frac{\mathrm{Z}}{\mathrm{z}_{0}}}$ & $\frac{2}{\left(1+\mathrm{v}_{\mathrm{H}} / \mathrm{v}_{\mathrm{i}}\right)}$ & $\frac{4}{\left(1+\mathrm{v}_{\mathrm{H}} / \mathrm{v}_{\mathrm{i}}\right)\left(1+\sqrt{\mathrm{v}_{\mathrm{H}}} / \mathrm{v}_{\mathrm{i}}\right)}$ \\
\hline 0.30 & 1.0000 & 1.0000 & 1.0000 \\
\hline 1.00 & 1.1402 & 0.9345 & 0.9038 \\
\hline 3.00 & 1.4142 & 0.8284 & 0.8013 \\
\hline 10.00 & 2.0000 & 0.6677 & 0.5523 \\
\hline 30.00 & 3.3166 & 0.4633 & 0.3285 \\
\hline
\end{tabular}

Table I. Reduction of growth with acceleration for current proportional to velocity and constant current. The tabulated factor is the reduction of exponential growth rate compared with that of a coasting beam. 

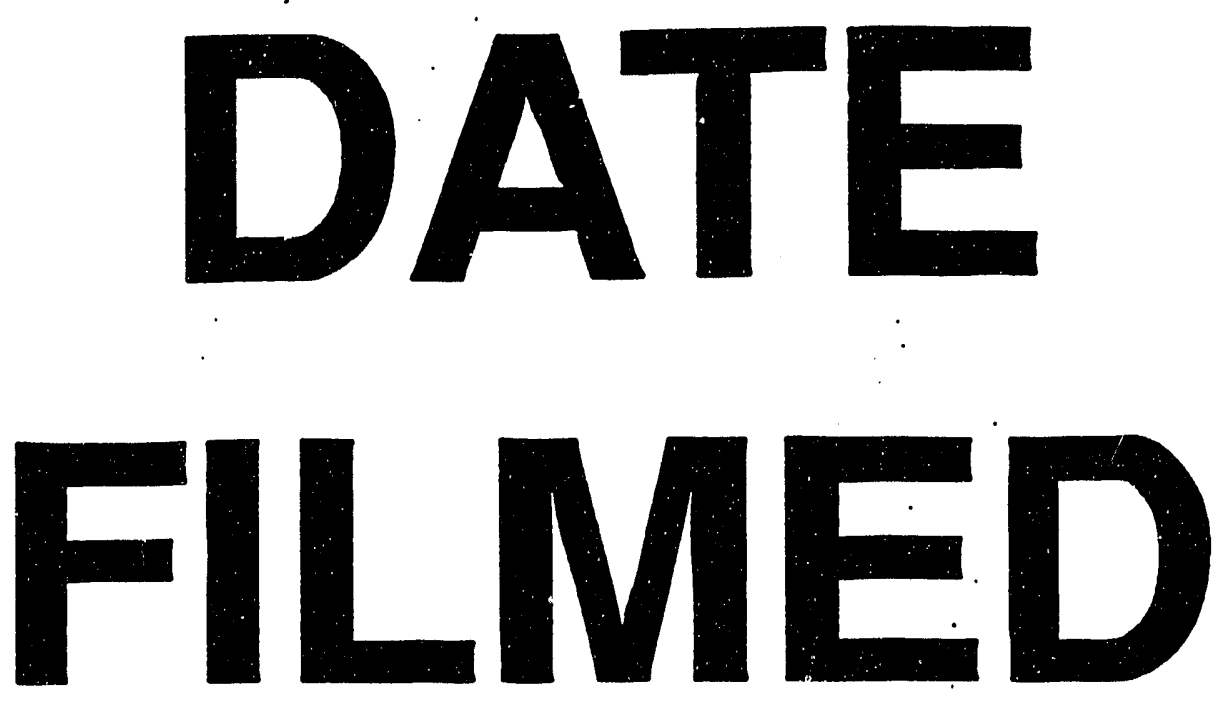

$10 / 20 / 93$
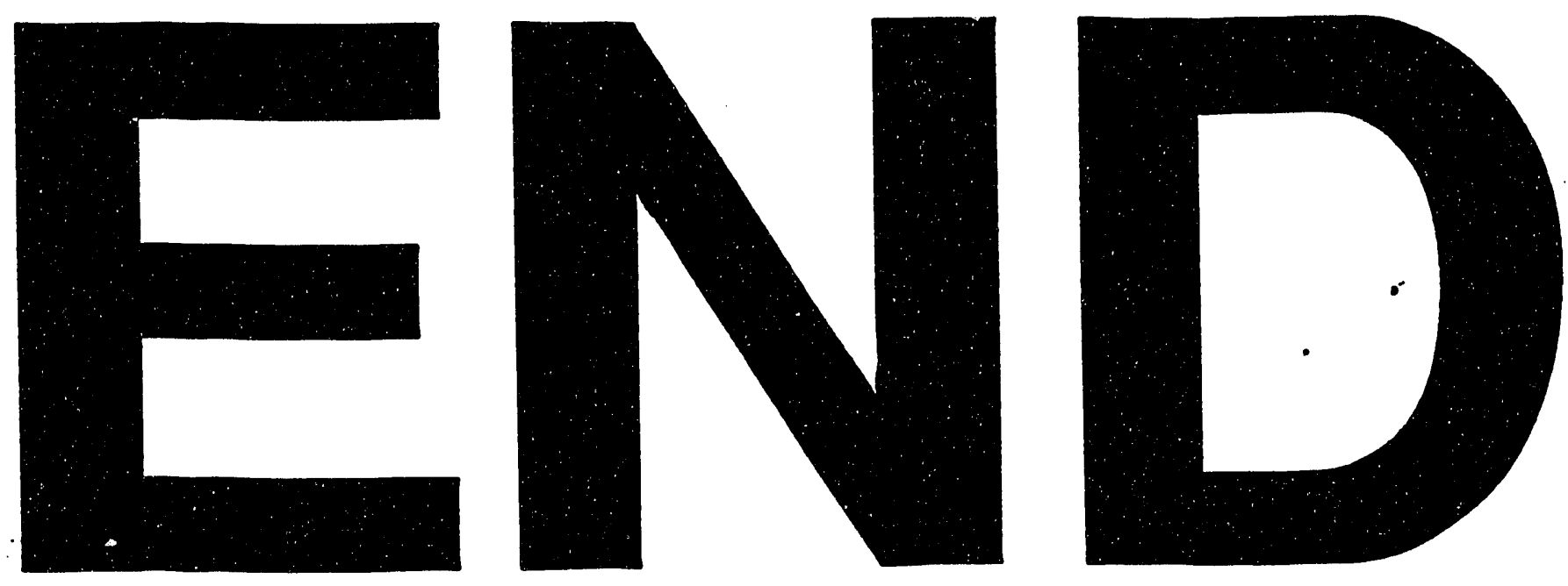
